JOURNAL OF THE

AMERICAN MATHEMATICAL SOCIETY

Volume 14, Number 2, Pages 471-486

S 0894-0347(00)00361-1

Article electronically published on December 13, 2000

\title{
BRAID GROUPS ARE LINEAR
}

\author{
STEPHEN J. BIGELOW
}

\section{INTRODUCTION}

The braid groups $B_{n}$ were originally introduced by Emil Artin in 1926. They have many interpretations, for example, as the group of geometric braids in $\mathbf{R}^{3}$, as the Artin group of type $A_{n}$, as the fundamental group of a certain hyperplane arrangement, as a subgroup of the automorphism group of a free group, and so on. In this paper we will use the interpretation of $B_{n}$ as the mapping class group of an $n$-times punctured disk.

A group is said to be linear if it admits a faithful representation into $\operatorname{GL}(m, \mathbf{R})$ for some natural number $m$. The question of whether braid groups are linear probably dates back to 1935 when Burau [Bur36] discovered an $n$-dimensional representation of $B_{n}$. For a long time this was thought to be a possible candidate for a faithful representation. A simple proof that it is faithful in the case $n=3$ gave some reason for optimism. However in 1991, Moody Moo91 showed that the Burau representation is not faithful for $n \geq 9$. This was later brought down to $n \geq 6$ and then $n \geq 5$ in the papers [LP93] and [Big99].

It therefore came as a pleasant surprise when Krammer Kra99. proved that another representation of the braid groups is faithful in the case $n=4$. The representation Krammer used is essentially the same as one used by Lawrence in Law90 to give a topological definition of a certain summand of the Jones representation. We call this representation the Lawrence-Krammer representation. In this paper, we prove the following.

Theorem 1.1. The Lawrence-Krammer representation of $B_{n}$ is faithful for all $n$.

This proves that all braid groups are linear. Our proof can be seen as a sort of converse to the construction of elements of the kernel of the Burau representation given in [Moo91, LP93] and Big99. Our methods are topological, and very different from the algebraic methods used by Krammer for the case $n=4$. Recently, Krammer [Kra00] extended his algebraic methods to obtain a new proof of the above theorem as well as some new ways to read information about a braid from its Lawrence-Krammer representation.

This paper represents the main result of the author's doctoral thesis at the University of California at Berkeley. The author thanks his advisor Professor Kirby, as well as Professors Casson, Jones, Stallings, and many others who thoroughly checked the arguments for accuracy and aesthetics. Thanks also to V. Turaev,

Received by the editors May 11, 2000 and, in revised form, October 30, 2000.

2000 Mathematics Subject Classification. Primary 20F36; Secondary 57M07, $20 \mathrm{C} 15$.

Key words and phrases. Braid group, linear, representation.

(C)2000 American Mathematical Society 
whose survey paper [Tur] filled some gaps in the exposition of an earlier draft of this paper.

1.1. Outline. In Section 1 we define a certain covering space $\tilde{C}$ of the configuration space $C$ of unordered pairs of distinct points in the $n$-times punctured disk. The Lawrence-Krammer representation is defined to be the induced action of the braid group $B_{n}$ on the second homology group of $\tilde{C}$.

In Section 2 we define forks and noodles. These are one-dimensional objects in the disk designed to represent elements of the second homology and cohomology of $\tilde{C}$. We define an intersection pairing between a noodle and a fork. We prove that if $F$ is a fork and $\sigma$ is an element of the kernel of the Lawrence-Krammer representation, then $F$ and $\sigma(F)$ have the same pairing with any fixed noodle.

In Section 3 we prove that the pairing between a noodle and a fork detects geometric intersection between the corresponding edges in the disk. We use this to show that a braid in the kernel of the Lawrence-Krammer representation must be trivial.

In Section 4 we give an explicit description of the Lawrence-Krammer representation in terms of the action of generators of $B_{n}$ on basis elements of $H_{2}(\tilde{C})$.

1.2. Definitions. Let $D$ be an oriented disk in the complex plane. Fix a set $P \subset D$ consisting of $n$ distinct points $p_{1}, \ldots, p_{n}$ in the interior of $D$. We will call these puncture points. Let $\mathcal{H}(D, P)$ be the group of all homeomorphisms $h: D \rightarrow D$ such that $h(P)=P$ and $h$ fixes $\partial D$ pointwise. Let $\mathcal{I}(D, P)$ be the group of all such homeomorphisms which are isotopic to the identity relative to $\partial D \cup P$. We define the braid group $B_{n}$ to be the group $\mathcal{H}(D, P) / \mathcal{I}(D, P)$. See Bir74 for other equivalent definitions of these groups and a good introduction to their basic properties.

Let $C$ denote the space of all unordered pairs of distinct points in $D \backslash P$. In other words,

$$
C=\frac{((D \backslash P) \times(D \backslash P)) \backslash\{(x, x)\}}{(x, y) \sim(y, x)} .
$$

Let $d_{1}$ and $d_{2}$ be distinct points in $\partial D$. Let $c_{0}=\left\{d_{1}, d_{2}\right\}$ be a basepoint for $C$. We now define a map $\phi$ from $\pi_{1}\left(C, c_{0}\right)$ to the free Abelian group with basis $\{q, t\}$. Let $\alpha$ be a closed curve in $C$ based at $c_{0}$ representing an element $[\alpha]$ of $\pi_{1}\left(C, c_{0}\right)$. We can write $\alpha$ in the form

$$
\alpha(s)=\left\{\alpha_{1}(s), \alpha_{2}(s)\right\}
$$

for some $\operatorname{arcs} \alpha_{1}$ and $\alpha_{2}$ in $D \backslash P$. Let

$$
a=\frac{1}{2 \pi i} \sum_{j=1}^{n}\left(\int_{\alpha_{1}} \frac{d z}{z-p_{j}}+\int_{\alpha_{2}} \frac{d z}{z-p_{j}}\right) .
$$

Let

$$
b=\frac{1}{\pi i} \int_{\alpha_{1}-\alpha_{2}} \frac{d z}{z} .
$$

Let $\phi([\alpha])=q^{a} t^{b}$.

This definition requires some explanation. If $\alpha_{1}$ and $\alpha_{2}$ are closed loops, then $a$ is the sum of the winding numbers of $\alpha_{1}$ and $\alpha_{2}$ around each of the puncture points, and $b$ is twice the winding number of $\alpha_{1}$ and $\alpha_{2}$ around each other. However $\alpha_{1}$ and $\alpha_{2}$ are not necessarily closed loops, but may "switch places". In this case, 
$a$ is the sum of the winding numbers of the closed loop $\alpha_{1} \alpha_{2}$ around each of the puncture points. Furthermore, $\alpha_{1}-\alpha_{2}$ satisfies

$$
\left(\alpha_{1}-\alpha_{2}\right)(1)=-\left(\alpha_{1}-\alpha_{2}\right)(0)
$$

which implies that $b$ is an odd integer.

Let $\tilde{C}$ be the covering space of $C$ whose fundamental group is the kernel of $\phi$. Fix a lift $\tilde{c}_{0}$ of $c_{0}$ to $\tilde{C}$. Let $\Lambda$ denote the ring $\mathbf{Z}\left[q^{ \pm 1}, t^{ \pm 1}\right]$. The homology group $H_{2}(\tilde{C})$ can be considered as a $\Lambda$-module, where $q$ and $t$ act by covering transformations.

Any homeomorphism $\sigma \in \mathcal{H}(D, P)$ induces a homeomorphism from $C$ to itself, also denoted $\sigma$. It is easy to check that $\sigma$ fixes $c_{0}$ and the induced action of $\sigma$ on $\pi_{1}\left(C, c_{0}\right)$ satisfies $\phi \sigma=\phi$. Thus $\sigma$ lifts uniquely to a map $\tilde{\sigma}$ from $\tilde{C}$ to itself which fixes $\tilde{c}_{0}$. Moreover, this lift $\tilde{\sigma}$ commutes with the covering transformations $q$ and $t$. It follows that the induced action $\tilde{\sigma}_{*}$ of $\tilde{\sigma}$ on $H_{2}(\tilde{C})$ is a $\Lambda$-module automorphism. The Lawrence-Krammer representation is the map from $B_{n}$ to $\operatorname{GL}\left(H_{2}(\tilde{C})\right)$ taking $[\sigma]$ to $\tilde{\sigma}_{*}$.

We will see in Section 4 how to embed $\operatorname{GL}\left(H_{2}(\tilde{C})\right)$ into $\operatorname{GL}\left(\left(\begin{array}{l}n \\ 2\end{array}\right), \mathbf{R}\right)$. We therefore obtain a faithful $\left(\begin{array}{l}n \\ 2\end{array}\right)$-dimensional representation of $B_{n}$ over $\mathbf{R}$.

In [Law90], Lawrence defines a representation by a similar construction using the space of ordered $k$-tuples of distinct points in $D \backslash P$. In the case $k=2$, the resulting representation is the same map from $B_{n}$ to $\operatorname{GL}\left(H_{2}(\tilde{C})\right)$ as defined above, except that $H_{2}(\tilde{C})$ is to be considered as a module over $\mathbf{Z}\left[q^{ \pm 1}, t^{ \pm 2}\right]$. As a result, the representation defined by Lawrence has degree $n(n-1)$, which is double that of the Lawrence-Krammer representation. The variable $\alpha$ in [Law90] corresponds to $t^{2}$ in this paper.

In Kra99, Krammer defines a representation of $B_{n}$ as the induced action on a module of formal $\Lambda$-linear combinations of forks modulo certain relations. These forks are of central importance in this paper, and will be defined in Section 2 Krammer's representation is the same as our Lawrence-Krammer representation except that the variable $t$ in [Kra99] corresponds to $-t$ in this paper.

1.3. Notation. Throughout this paper, $I$ will denote the interval $[0,1]$.

If $\alpha$ and $\beta$ are arcs in $D \backslash P$ such $\alpha(s) \neq \beta(s)$ for all $s \in I$, then denote by $\{\alpha, \beta\}$ the arc in $C$ given by

$$
\{\alpha, \beta\}(s)=\{\alpha(s), \beta(s)\} .
$$

If $y$ is a point in $D \backslash P$ and $\alpha$ is an arc in $D \backslash(P \cup\{y\})$, then denote by $\{\alpha, y\}$ the arc in $C$ given by

$$
\{\alpha, y\}(s)=\{\alpha(s), y\}
$$

The same arc can also be denoted by $\{y, \alpha\}$.

If $g$ and $h$ are elements of a group, then we use the notation

$$
g^{h}=h^{-1} g h
$$

and

$$
[g, h]=g^{-1} h^{-1} g h .
$$

Braids compose from right to left. Arcs compose from left to right. 


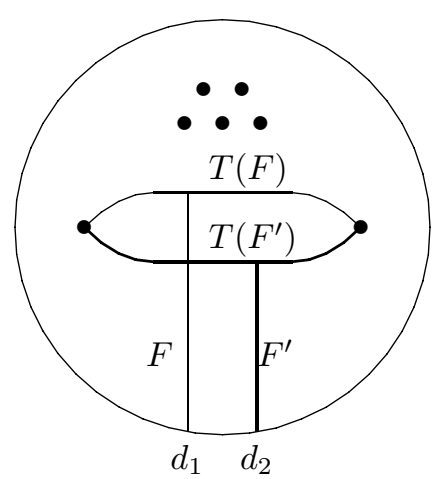

Figure 1. A fork $F$ and a parallel copy $F^{\prime}$.

\section{FORKS AND NOODLES}

In this section we define forks and noodles and a pairing between them. The idea of using a fork to represent an element of $H_{2}(\tilde{C})$ is due to Krammer Kra99.

A fork is an embedded tree $F \subset D$ with four vertices $d_{1}, p_{i}, p_{j}$ and $z$ such that $F \cap \partial D=\left\{d_{1}\right\}, F \cap P=\left\{p_{i}, p_{j}\right\}$, and all three edges have $z$ as a vertex. The edge containing $d_{1}$ is called the handle of $F$. The union of the other two edges is a single edge, which we call the tine edge of $F$ and denote by $T(F)$. We orient $T(F)$ in such a way that the handle of $F$ lies to the right of $T(F)$.

For a given fork $F$ we can define a parallel copy of $F$ to be an embedded tree $F^{\prime}$ as shown in Figure 1. The five puncture points at the top of the figure may be replaced by any number, and any orientation-preserving self-homeomorphism may be applied to the entire disk. The tine edge $T\left(F^{\prime}\right)$ of $F^{\prime}$ is defined analogously to that of $F$.

For any fork $F$, we define a surface $\tilde{\Sigma}(F)$ in $\tilde{C}$ as follows. Let $F^{\prime}$ be a parallel copy of $F$. Let $z$ be the vertex contained in all three edges of $F$, and let $z^{\prime}$ be the vertex contained in all three edges of $F^{\prime}$. Define a surface $\Sigma(F)$ in $C$ to be the set of all points which can be written in the form $\{x, y\}$, where $x \in T(F) \backslash P$ and $y \in T\left(F^{\prime}\right) \backslash P$. Let $\beta_{1}$ be an arc from $d_{1}$ to $z$ along the handle of $F$ and let $\beta_{2}$ be an arc from $d_{2}$ to $z^{\prime}$ along the handle of $F^{\prime}$. Let $\tilde{\beta}$ be the lift of $\left\{\beta_{1}, \beta_{2}\right\}$ to $\tilde{C}$ beginning at $\tilde{c}_{0}$. Let $\tilde{\Sigma}(F)$ be the lift of $\Sigma(F)$ to $\tilde{C}$ which contains $\tilde{\beta}(1)$.

There is an obvious homeomorphism from $\Sigma(F)$ to the interior of the square $T(F) \times T\left(F^{\prime}\right)$. The orientations on $T(F)$ and $T\left(F^{\prime}\right)$ give rise to an orientation on $T(F) \times T\left(F^{\prime}\right)$, and hence on $\Sigma(F)$. This lifts to an orientation on $\tilde{\Sigma}(F)$.

A noodle is an embedded edge $N \subset D \backslash P$ with endpoints $d_{1}$ and $d_{2}$. Orient $N$ so that it goes from $d_{1}$ to $d_{2}$. For a given noodle $N$ we define the surface $\Sigma(N)$ to be the set of points $\{x, y\} \in C$ such that $x$ and $y$ are distinct points in $N$. Let $\tilde{\Sigma}(N)$ be the lift of $\Sigma(N)$ to $\tilde{C}$ which contains $\tilde{c}_{0}$.

There is an obvious homeomorphism from $\Sigma(N)$ to the subset of $N \times N$ consisting of those points $(x, y)$ such that $d_{1}$ is closer to $x$ than it is to $y$ along $N$. The orientation on $N$ gives rise to an orientation on $N \times N$, and hence on $\Sigma(N)$. This lifts to an orientation on $\tilde{\Sigma}(N)$.

2.1. A pairing. Let $N$ be a noodle and let $F$ be a fork. We use the surfaces $\tilde{\Sigma}(N)$ and $\tilde{\Sigma}(F)$ to define an element $\langle N, F\rangle$ of $\Lambda$ as follows. 
If necessary, apply a preliminary isotopy so that $T(F)$ intersects $N$ transversely. Let $z_{1}, \ldots, z_{l}$ denote the points of intersection between $N$ and $T(F)$. Let $F^{\prime}$ be a parallel copy of $F$ such that $T\left(F^{\prime}\right)$ intersects $N$ transversely at $z_{1}^{\prime}, \ldots, z_{l}^{\prime}$, where $z_{i}$ and $z_{i}^{\prime}$ are joined by a short arc in $N$ which lies in the narrow strip bounded by $T(F)$ and $T\left(F^{\prime}\right)$. For $i, j=1, \ldots, l$, there exists a unique monomial $m_{i, j}=q^{a_{i, j}} t^{b_{i, j}}$ such that $m_{i, j} \tilde{\Sigma}(N)$ intersects $\tilde{\Sigma}(F)$ at a point lying over $\left\{z_{i}, z_{j}^{\prime}\right\} \in C$. Let $\epsilon_{i, j}$ be the sign of that intersection. Then we define the pairing between $N$ and $F$ as

$$
\langle N, F\rangle=\sum_{i=1}^{l} \sum_{j=1}^{l} \epsilon_{i, j} m_{i, j} .
$$

We need to prove that this does not depend on our choice of preliminary isotopy of $F$. First we describe how to explicitly compute the pairing.

We compute $m_{i, j}$ as follows. Define the following embedded arcs in $D \backslash P$.

- $\alpha_{1}$ from $d_{1}$ to $z$ along the handle of $F$,

- $\alpha_{2}$ from $d_{2}$ to $z^{\prime}$ along the handle of $F^{\prime}$,

- $\beta_{1}$ from $z$ to $z_{i}$ along $T(F)$,

- $\beta_{2}$ from $z^{\prime}$ to $z_{j}^{\prime}$ along $T\left(F^{\prime}\right)$,

- $\gamma_{1}$ from $z_{i}$ to $d_{k}$ along $N$, where $k \in\{1,2\}$ is such that $\gamma_{1}$ does not pass through $z_{j}^{\prime}$,

- $\gamma_{2}$ from $z_{j}^{\prime}$ to $d_{k^{\prime}}$ along $N$, where $k^{\prime} \in\{1,2\}$ is such that $\gamma_{2}$ does not pass through $z_{i}$.

Consider the following arc in $C$ :

$$
\delta_{i, j}=\left\{\alpha_{1}, \alpha_{2}\right\}\left\{\beta_{1}, \beta_{2}\right\}\left\{\gamma_{1}, \gamma_{2}\right\} .
$$

Let $\tilde{\delta}_{i, j}$ be the lift of $\delta_{i, j}$ beginning at $\tilde{c}_{0}$. This goes first to $\tilde{\Sigma}(F)$, then to the lift of $\left\{z_{i}, z_{j}^{\prime}\right\}$ which lies in $\tilde{\Sigma}(F) \cap m_{i, j} \tilde{\Sigma}(N)$, and finally to $m_{i, j} \tilde{c}_{0}$. Thus $\tilde{\delta}_{i, j}$ goes from $\tilde{c}_{0}$ to $m_{i, j} \tilde{c}_{0}$, so

$$
m_{i, j}=\phi\left(\delta_{i, j}\right) .
$$

We now compute $\epsilon_{i, j}$. This is the sign of the intersection of $\Sigma(N)$ with $\Sigma(F)$ at the point $\left\{z_{i}, z_{j}^{\prime}\right\}$. A direct computation using our choice of orientations for $\Sigma(N)$ and $\Sigma(F)$ shows that $\epsilon_{i, j}=-a b c$, where

- $a$ is the sign of the intersection of $N$ and $T(F)$ at $z_{i}$,

- $b$ is the sign of the intersection of $N$ and $T\left(F^{\prime}\right)$ at $z_{j}^{\prime}$,

- $c$ is 1 if $d_{1}$ is closer to $z_{i}$ than it is to $z_{j}^{\prime}$ along $N$, and -1 otherwise.

Now $b_{i, j}$ is odd if and only if the two points switch places in the path $\delta_{i, j}$. This happens if and only if $d_{1}$ is closer to $z_{j}^{\prime}$ than it is to $z_{i}$ along $N$. Thus $c=(-1)^{b_{i, j}}$. Similarly, $b_{i, i}$ is odd if and only if $d_{1}$ is closer to $z_{i}^{\prime}$ than it is to $z_{i}$ along $N$. This happens if and only if the intersection between $N$ and $T(F)$ at $z_{i}$ is negative. Thus $a=(-1)^{b_{i, i}}$. Similarly $b=(-1)^{b_{j, j}}$. We conclude that

$$
\epsilon_{i, j}=-(-1)^{b_{i, i}+b_{j, j}+b_{i, j}} \text {. }
$$

The following will be useful later.

Lemma 2.1. $a_{i, j}=\left(a_{i, i}+a_{j, j}\right) / 2$.

Proof. For $i=1, \ldots, l$ let $\xi_{i}$ be the arc in $D \backslash P$ which goes from $d_{1}$ to $z_{i}$ in $F$ and then back to $d_{1}$ in $N$. Let $a_{i}$ be the sum of the winding numbers of $\xi_{i}$ around each of the puncture points. Let $\xi$ be the arc in $D \backslash P$ which goes from $d_{1}$ to $d_{2}$ along $N$ 
and then back to $d_{1}$ counterclockwise around $\partial D$. Let $a$ be the sum of the winding numbers of $\xi$ around each of the puncture points. I claim that $a_{i, j}=a_{i}+a_{j}+a$.

Let $\delta_{i, j}=\left\{\alpha_{1}, \alpha_{2}\right\}\left\{\beta_{1}, \beta_{2}\right\}\left\{\gamma_{1}, \gamma_{2}\right\}$ be as above. If $\alpha_{1} \beta_{1} \gamma_{1}$ and $\alpha_{2} \beta_{2} \gamma_{2}$ are closed arcs, then $\alpha_{1} \beta_{1} \gamma_{1}=\xi_{i}$ and $\alpha_{2} \beta_{2} \gamma_{2}$ is freely homotopic to $\xi_{j} \xi$. On the other hand, if $\alpha_{1} \beta_{1} \gamma_{1} \alpha_{2} \beta_{2} \gamma_{2}$ is a single closed loop, then it is freely homotopic to $\xi_{i} \xi \xi_{j}$. In either case, the exponent of $q$ in $\phi\left(\delta_{i, j}\right)$ is the sum of the winding numbers of $\xi_{i}, \xi_{j}$ and $\xi$ around each of the puncture points. Thus $a_{i, j}=a_{i}+a_{j}+a$.

Similarly, $a_{i, i}=2 a_{i}+a$ and $a_{j, j}=2 a_{j}+a$. Thus $a_{i, j}=\left(a_{i, i}+a_{j, j}\right) / 2$.

2.2. The pairing is well defined. We now prove the following lemma.

Lemma 2.2. Suppose $F_{1}$ and $F_{2}$ are isotopic relative to $\partial D \cup P$ such that $T\left(F_{1}\right)$ and $T\left(F_{2}\right)$ intersect $N$ transversely. Then $\left\langle N, F_{1}\right\rangle=\left\langle N, F_{2}\right\rangle$.

This is a special case of Lemma 2.3 but it is easy enough to prove directly.

A generic isotopy of $F_{1}$ can be split into a finite sequence of moves of two types:

- isotopies which keep $T\left(F_{1}\right)$ transverse to $N$,

- isotopies which push a small subarc of $T\left(F_{1}\right)$ across a subarc of $N$, either adding or removing a pair of points from the set $N \cap T\left(F_{1}\right)$.

A move of the first type has no effect on the computation of $\left\langle N, F_{1}\right\rangle$. As for the second type, it suffices to consider the case in which a pair of intersection points $z_{l+1}$ and $z_{l+2}$ is added to $N \cap T\left(F_{1}\right)=\left\{z_{1}, \ldots, z_{l}\right\}$.

There are now two cases to consider. First, suppose $z_{l+1}^{\prime}$ and $z_{l+2}^{\prime}$ lie between $z_{l+1}$ and $z_{l+2}$ on $N$. It follows from the definitions and the discussion above that

$$
m_{i, l+1}=m_{i, l+2}
$$

and

$$
\epsilon_{i, l+1}=-\epsilon_{i, l+2}
$$

for any $i=1, \ldots, l+2$. Also,

$$
m_{l+1, j}=m_{l+2, j}
$$

and

$$
\epsilon_{l+1, j}=-\epsilon_{l+2, j}
$$

for $j=1, \ldots, l$. Thus we have complete cancellation of the terms $\epsilon_{i, j} m_{i, j}$ for which either $i$ or $j$ or both lie in $\{l+1, l+2\}$. Therefore the computation of $\left\langle N, F_{1}\right\rangle$ gives the same answer before and after the move.

A similar argument works if $z_{l+1}$ and $z_{l+2}$ lie between $z_{l+1}^{\prime}$ and $z_{l+2}^{\prime}$ on $N$. In that case,

$$
\epsilon_{l+1, j} m_{l+2, j}=-\epsilon_{l+2, j} m_{l+2, j}
$$

for $j=1, \ldots, l+2$, and

$$
\epsilon_{i, l+1} m_{i, l+1}=-\epsilon_{i, l+2} m_{i, l+2}
$$

for $i=1, \ldots, l$. 
2.3. The Basic Lemma. We now prove the following lemma.

Lemma 2.3 (The Basic Lemma). If $[\sigma]$ lies in the kernel of the Lawrence-Krammer representation, then

$$
\langle N, F\rangle=\langle N, \sigma(F)\rangle
$$

for every noodle $N$ and fork $F$.

Let $[\sigma]$ be an element of the kernel of the Lawrence-Krammer representation. We can assume that the tine edges of $F$ and $\sigma(F)$ intersect $N$ transversely. Then $\Sigma(F)$ intersects $\Sigma(N)$ transversely. Thus $\tilde{\Sigma}(F)$ intersects any lift $q^{a} t^{b} \tilde{\Sigma}(N)$ transversely. Let $\left(q^{a} t^{b} \tilde{\Sigma}(N), \tilde{\Sigma}(F)\right)$ denote the algebraic intersection number between these surfaces. Then an equivalent definition of the pairing between $N$ and $F$ is as follows:

$$
\langle N, F\rangle=\sum_{a, b \in \mathbf{Z}}\left(q^{a} t^{b} \tilde{\Sigma}(N), \tilde{\Sigma}(F)\right) q^{a} t^{b} .
$$

We must show that $\tilde{\Sigma}(F)$ and $\sigma(\tilde{\Sigma}(F))$ have the same algebraic intersection number with $q^{a} t^{b} \tilde{\Sigma}(N)$. The difficulty comes from the fact that the surfaces $\tilde{\Sigma}(N)$ and $\tilde{\Sigma}(F)$ are not closed, but are only properly embedded. The algebraic intersection between two properly embedded surfaces can be problematic, since it may be possible to push intersections off to infinity. To solve this problem, we will prove the existence of an immersed closed surface $\tilde{\Sigma}_{2}(F)$ which is equal to $(1-q)^{2}(1+q t) \tilde{\Sigma}(F)$ outside a small neighborhood of the puncture points.

Let $F$ be a fork. Let the endpoints of $T(F)$ be $p_{i}$ and $p_{j}$. Let $\nu\left(p_{i}\right)$ and $\nu\left(p_{j}\right)$ be disjoint $\epsilon$-neighborhoods of $p_{i}$ and $p_{j}$ respectively such that $\nu\left(p_{k}\right) \cap P=\left\{p_{k}\right\}$ for $k=i, j$. Let $U$ be the set of $\{x, y\} \in C$ such that at least one of $x$ and $y$ lies in $\nu\left(p_{i}\right) \cup \nu\left(p_{j}\right)$. Fix a basepoint $u_{0}=\left\{u_{1}, u_{2}\right\} \in U$, where $u_{1} \in \nu\left(p_{i}\right)$ and $u_{2} \in \nu\left(p_{j}\right)$. Let $\tilde{U}$ be the pre-image of $U$ in $\tilde{C}$. This is a connected covering space of $U$. Choose a lift $\tilde{u}_{0}$ of $u_{0}$ to $\tilde{C}$.

Now $\tilde{\Sigma}(F)$ represents an element of the relative homology group $H_{2}(\tilde{C}, \tilde{U})$. Our goal is to find a corresponding element of the homology group $H_{2}(\tilde{C})$. We start by analyzing $\pi_{1}\left(U, u_{0}\right)$. This will give us information about the subgroup $\pi_{1}\left(\tilde{U}, \tilde{u}_{0}\right)$. This in turn will give us information about $H_{1}(\tilde{U})$. Finally, we will use the long exact sequence of relative homology to obtain the required element of $H_{2}(\tilde{C})$.

Using the arcs shown in Figure 2, we define the following elements of $\pi_{1}\left(U, u_{0}\right)$ :

$$
\begin{aligned}
& a_{1}=\left\{\gamma_{1}, u_{2}\right\}, \\
& a_{2}=\left\{u_{1}, \gamma_{2}\right\}, \\
& b_{1}=\left\{\alpha_{1}, \beta_{1} \beta_{2} \beta_{3}\right\}\left\{\alpha_{2} \alpha_{3}, u_{1}\right\} \\
& b_{2}=\left\{\alpha_{1} \alpha_{2} \alpha_{3}, \beta_{1}\right\}\left\{u_{2}, \beta_{2} \beta_{3}\right\} .
\end{aligned}
$$

Note that $b_{1}$ and $b_{2}$ are homotopic in $C$, but not in $U$.

The following relations hold in $\pi_{1}\left(U, u_{0}\right)$ :

$$
\begin{aligned}
{\left[a_{1}, a_{2}\right] } & =1 \\
{\left[a_{1}, b_{1} a_{1} b_{1}\right] } & =1 \\
{\left[a_{2}, b_{2} a_{2} b_{2}\right] } & =1
\end{aligned}
$$




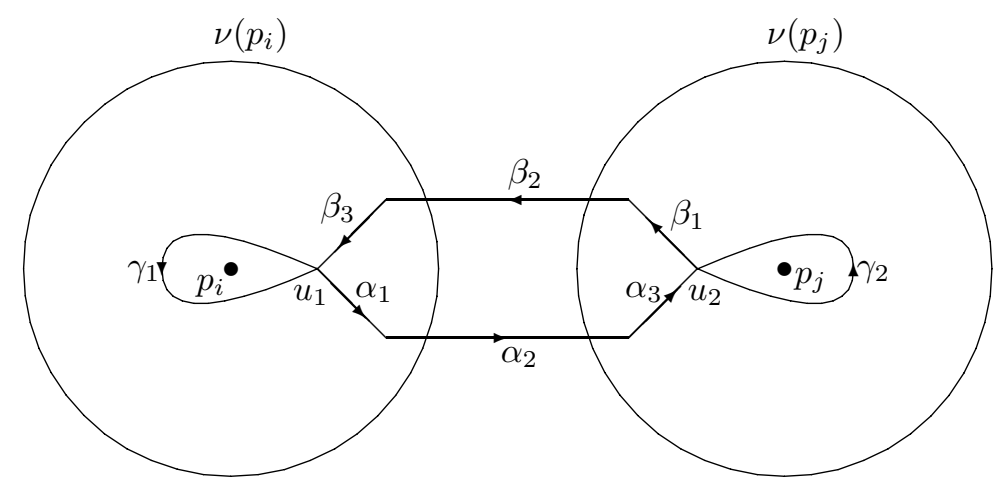

Figure 2. Some $\operatorname{arcs}$ in $D$.

The first of these is obvious. The second follows from the fact that $b_{1} a_{1} b_{1}$ is equal in $\pi_{1}\left(U, u_{0}\right)$ to $\left\{u_{1}, \delta\right\}$, where $\delta$ is a curve based at $u_{2}$ which passes counterclockwise around $p_{1}$ and $u_{1}$. The third follows by a similar argument.

We now analyze $\pi_{1}\left(\tilde{U}, \tilde{u}_{0}\right)$, considered as a subgroup of $\pi_{1}\left(U, u_{0}\right)$. Let $i: U \rightarrow C$ be the inclusion map and let $i_{*}$ be the induced map on fundamental groups. Then $\pi_{1}\left(\tilde{U}, \tilde{u}_{0}\right)$ is the kernel of the map $\phi i_{*}$. We define the following elements of $\pi_{1}\left(\tilde{U}, \tilde{u}_{0}\right)$ :

$$
\begin{aligned}
a & =a_{2}^{-1} a_{1}, \\
b & =b_{2}^{-1} b_{1}, \\
c & =a_{1}^{-1} b_{1}^{-1} a_{1} b_{1}, \\
d & =a_{2}^{-1} b_{2}^{-1} a_{2} b_{2} .
\end{aligned}
$$

If $x \in \pi_{1}\left(\tilde{U}, \tilde{u}_{0}\right)$ and $y \in \pi_{1}\left(U, u_{0}\right)$, then the conjugate $x^{y}=y^{-1} x y$ is also an element of $\pi_{1}\left(\tilde{U}, \tilde{u}_{0}\right)$. The following relations hold in $\pi_{1}\left(\tilde{U}, \tilde{u}_{0}\right)$ :

$$
\begin{aligned}
a^{a_{1}} & =a, \\
c^{b_{1} a_{1}} c & =1, \\
d^{b_{2} a_{2}} d & =1, \\
d b a^{b_{1}} & =a b^{a_{1}} c .
\end{aligned}
$$

To see this, rewrite these relations in terms of $a_{1}, a_{2}, b_{1}$ and $b_{2}$. The first three translate into equations (2) to (4). The fourth translates into a trivial identity.

If $x \in \pi_{1}\left(\tilde{U}, \tilde{u}_{0}\right)$, let $[x]$ denote the corresponding element of $H_{1}(\tilde{U})$. Note that if $x \in \pi_{1}\left(\tilde{U}, \tilde{u}_{0}\right)$ and $y \in \pi_{1}\left(U, u_{0}\right)$, then $\left[x^{y}\right]=\phi(y)^{-1}[x]$. The relations given in equations (5) to (8) give rise to the following relations in $H_{1}(\tilde{U})$ :

$$
\begin{aligned}
\left(q^{-1}-1\right)[a] & =0, \\
\left(q^{-1} t^{-1}+1\right)[c] & =0, \\
\left(q^{-1} t^{-1}+1\right)[d] & =0, \\
\left(q^{-1}-1\right)[b] & =\left(t^{-1}-1\right)[a]-[c]+[d] .
\end{aligned}
$$

Combining these relations, we obtain

$$
(1-q)^{2}(1+q t)[b]=0 .
$$


Let $[\tilde{\Sigma}(F)]$ be the element of $H_{2}(\tilde{C}, \tilde{U})$ represented by $\tilde{\Sigma}(F)$. The long exact sequence of relative homology gives us the following exact sequence of $\Lambda$-modules:

$$
H_{2}(\tilde{C}) \stackrel{j_{*}}{\rightarrow} H_{2}(\tilde{C}, \tilde{U}) \stackrel{\partial}{\rightarrow} H_{1}(\tilde{U}) .
$$

But $\partial[\tilde{\Sigma}(F)]=[b]$. It follows that

$$
(1-q)^{2}(1+q t)[\tilde{\Sigma}(F)]=j_{*}\left[\tilde{\Sigma}_{2}(F)\right]
$$

for some $\left[\tilde{\Sigma}_{2}(F)\right] \in H_{2}(\tilde{C})$ represented by some immersed closed surface $\tilde{\Sigma}_{2}(F)$. Then $\tilde{\Sigma}_{2}(F)$ is setwise equal to $(1-q)^{2}(1+q t) \tilde{\Sigma}(F)$ outside $U$.

Let $N$ be a noodle which intersects $T(F)$ transversely. Choose $\nu\left(p_{i}\right)$ and $\nu\left(p_{j}\right)$ small enough so as not to intersect $N$. Then

$$
(1-q)^{2}(1+q t)\langle N, F\rangle=\sum_{a, b \in \mathbf{Z}}\left(q^{a} t^{b} \tilde{\Sigma}(N), \tilde{\Sigma}_{2}(F)\right) q^{a} t^{b} .
$$

Now $\sigma$ is an element of the kernel of the Lawrence-Krammer representation, so acts as the identity on $H_{2}(\tilde{C})$. Thus the surfaces $\tilde{\Sigma}_{2}(F)$ and $\sigma\left(\tilde{\Sigma}_{2}(F)\right)$ represent the same element of homology, so have the same algebraic intersection with any $q^{a} t^{b} \tilde{\Sigma}(N)$. Since $\Lambda$ is a domain, it follows that $\langle N, \sigma(F)\rangle=\langle N, F\rangle$.

2.4. Alternative proofs. There are many possible approaches to proving the Basic Lemma. The proof given above is a compromise of sorts, since it proves the existence of an appropriate element of $H_{2}(\tilde{C})$, but does so in a non-constructive way.

It is possible to give a more constructive proof which uses an explicit computation of $H_{2}(\tilde{C})$. One obtains a concrete description of an immersed genus two surface which can be seen to be the same as $(1-q)^{2}(1+q t) \tilde{\Sigma}(F)$ away from the puncture points. This is perhaps best done in private, since the details are only convincing to the person who figures them out. Some details of a computation of $H_{2}(\tilde{C})$ will be given in Section 4. See also Law90, where Lawrence computes the middle homology of a covering space of the space of ordered $k$-tuples of distinct points in the $n$-times punctured disk, where $k$ can be any positive integer.

It is tempting to seek a less constructive proof which makes no reference to $\tilde{\Sigma}_{2}(F)$. It is intuitively obvious that the problem of pushing intersections off to infinity does not arise in the context of forks and noodles. However this line of reasoning runs into some technical difficulties which I feel distract from the true nature of the problem at hand. A proof that $B_{n}$ acts faithfully on $H_{2}(\tilde{C})$ should refer to an element of $H_{2}(\tilde{C})$.

It is possible to prove that braid groups are linear without reference to $C$, let alone $H_{2}(\tilde{C})$. The Lawrence-Krammer representation can be defined to be the action of $B_{n}$ on a $\Lambda$-module consisting of formal linear combinations of forks subject to certain relations, as described by Krammer in Kra99. The pairing $\langle N, F\rangle$ can be defined solely in terms of winding numbers. One must check that this pairing respects the relations between forks. The Basic Lemma then follows immediately. The rest of the proof that the Lawrence-Krammer representation is faithful proceeds virtually unchanged.

\section{The RePRESEntation IS Faithful}

In this section, we prove that the Lawrence-Krammer representation is faithful. The key step is to prove that the pairing $\langle N, F\rangle$ detects geometric intersection 
between $N$ and $T(F)$. That is, $\langle N, F\rangle$ is zero if and only if $T(F)$ can be isotoped off $N$ relative to $\partial D \cup P$.

We will use the following lemma from the basic theory of curves on surfaces.

Lemma 3.1. Let $\alpha$ and $\beta$ be simple closed curves in an orientable surface $\Sigma$ which intersect transversely. The following are equivalent.

- $\alpha$ is isotopic to a simple closed curve which intersects $\beta$ at fewer points,

- $\alpha$ and $\beta$ cobound a "digon", that is, an embedded disk in $\Sigma$ whose boundary consists of one subarc of $\alpha$ and one subarc of $\beta$.

A proof can be found in [FLP91, Proposition 3.10], or [PR99, Proposition 3.7].

This lemma as stated does not apply to the case of a noodle and the tine edge of a fork, since these are embedded edges and not simple closed curves. However these edges can be easily extended to simple closed curves by attaching handles to the surface $D \backslash P$. We will therefore use the above lemma in the context of noodles and forks without further comment.

Note that if $\alpha$ and $\beta$ cobound a digon, then it is easy to show that they cobound an "innermost digon" whose interior is disjoint from $\alpha \cup \beta$.

3.1. The Key Lemma. We now prove the following lemma.

Lemma 3.2 (The Key Lemma). Let $N$ be a noodle and let $F$ be a fork. Then $\langle N, F\rangle=0$ if and only if $T(F)$ is isotopic relative to $\partial D \cup P$ to an arc which is disjoint from $N$.

Let $N$ be a noodle and let $F$ be a fork. By applying a preliminary isotopy, we can assume that $T(F)$ intersects $N$ transversely at $z_{1}, \ldots, z_{l}$, where $l$ is the minimal possible number of points of intersection. Let $F^{\prime}$ be a parallel copy of $F$ such that $T\left(F^{\prime}\right)$ intersects $N$ transversely at $z_{1}^{\prime}, \ldots, z_{l}^{\prime}$, where $z_{i}$ and $z_{i}^{\prime}$ are joined by a short arc in $N$ which lies in the narrow strip bounded by $T(F)$ and $T\left(F^{\prime}\right)$. Let $m_{i, j}=q^{a_{i, j}} t^{b_{i, j}}$ be such that $m_{i, j} \tilde{\Sigma}(N)$ intersects $\tilde{\Sigma}(F)$ at a point lying over $\left\{z_{i}, z_{j}^{\prime}\right\} \in C$. Let $\epsilon_{i, j}$ be the sign of that intersection. Recall

$$
\langle N, F\rangle=\sum_{i=1}^{l} \sum_{j=1}^{l} \epsilon_{i, j} m_{i, j} .
$$

If $l=0$, then clearly $\langle N, F\rangle=0$. We now assume $l>0$ and show that $\langle N, F\rangle \neq 0$. We use the following lexicographic ordering on the set of monomials $q^{a} t^{b}$.

Definition 3.3. We say $q^{a} t^{b} \leq q^{a^{\prime}} t^{b^{\prime}}$ if and only if either

- $a<a^{\prime}$, or

- $a=a^{\prime}$ and $b \leq b^{\prime}$.

For $i, j \in\{1, \ldots, l\}$ we say that $m_{i, j}$ is maximal if $m_{i, j} \geq m_{i^{\prime}, j^{\prime}}$ for all $i^{\prime}, j^{\prime} \in$ $\{1, \ldots, l\}$.

Claim 3.4. If $m_{i, j}$ is maximal, then $m_{i, i}=m_{j, j}=m_{i, j}$.

Recall equation (1), which states that $\epsilon_{i, j}=-(-1)^{b_{i, i}+b_{j, j}+b_{i, j}}$. The above claim implies that if $m_{i, j}$ is maximal, then $\epsilon_{i, j}=-(-1)^{b_{i, j}}$. Thus all maximal monomials occur with the same sign in equation (9). Therefore $\langle N, F\rangle$ cannot equal zero. It remains only to prove the claim.

Suppose $m_{i, j}$ is maximal. Then $a_{i, j}$ is maximal among all the integers $a_{i^{\prime}, j^{\prime}}$. By Lemma 2.1 it follows that $a_{i, i}=a_{j, j}=a_{i, j}$. 
We now show that $b_{i, i}=b_{i, j}$. Since $m_{i, j}$ is maximal and $a_{i, i}=a_{i, j}$, it follows that $b_{i, i} \leq b_{i, j}$. Suppose, seeking a contradiction, that $b_{i, i}<b_{i, j}$. Let $\alpha$ be an embedded arc from $z_{i}^{\prime}$ to $z_{j}^{\prime}$ along $T\left(F^{\prime}\right)$. Let $\beta$ be an embedded arc from $z_{j}^{\prime}$ to $z_{i}^{\prime}$ along $N$.

If $\beta$ does not pass through the point $z_{i}$, let $w$ be the winding number of $\alpha \beta$ around $z_{i}$. I claim that $b_{i, j}-b_{i, i}=2 w$. To see this, consider the lift of $\left\{z_{i}, \alpha \beta\right\}$ to $\tilde{C}$ which starts in $\tilde{\Sigma}(F)$. This goes from $m_{i, i} \tilde{\Sigma}(N)$ to $m_{i, j} \tilde{\Sigma}(N)$. Thus $m_{i, j} m_{i, i}^{-1}=$ $\phi\left(\left\{z_{i}, \alpha \beta\right\}\right)$. Taking the exponent of $t$ in both sides gives the required equality.

If $\beta$ does pass through $z_{i}$, first modify $\beta$ in a small neighborhood of $z_{i}$ so that $z_{i}$ lies to its left, then let $w$ be the winding number of $\alpha \beta$ around $z_{i}$. I claim that $1+b_{i, j}-b_{i, i}=2 w$. We use a similar argument to that of the previous case. However $\beta$ deviates from $N$ to pass around $z_{i}$ in a counterclockwise (positive) direction. As a result, $\left\{z_{i}, \alpha \beta\right\}$ lifts to an arc from $m_{i, i} \tilde{\Sigma}(N)$ to $t m_{i, j} \tilde{\Sigma}(N)$. The extra factor of $t$ accounts for the addition of one to the left-hand side of the equation.

In either case, our assumption that $b_{i, i}<b_{i, j}$ implies that $w$ is greater than zero.

Let $D_{1}=D \backslash\left\{z_{i}\right\}$. Let $\pi: \tilde{D}_{1} \rightarrow D_{1}$ be the universal (infinite cyclic) cover. Let $\tilde{\alpha}$ be a lift of $\alpha$ to $\tilde{D}_{1}$. Let $\tilde{\beta}$ be the lift of $\beta$ to $\tilde{D}_{1}$ which starts at $\tilde{\alpha}(1)$. Let $\gamma$ be a loop in $D_{1}$ based at $z_{i}^{\prime}$ which winds $w$ times around $z_{i}$ in the clockwise (negative) direction, is null-homotopic in $D \backslash P$, and intersects $\alpha$ and $\beta$ only at its endpoints. Let $\tilde{\gamma}$ be the lift of $\gamma$ to an arc from $\tilde{\beta}(1)$ to $\tilde{\alpha}(0)$. Choose $\gamma$ so that $\tilde{\gamma}$ is an embedded arc.

Let $\tilde{z}_{k}^{\prime}$ be the first point on $\tilde{\alpha}$ which intersects $\tilde{\beta}$ (possibly $\tilde{\alpha}(1)$ ). This must be a lift of $z_{k}^{\prime}$ for some $k=1, \ldots, l$. Let $\tilde{\alpha}^{\prime}$ be the initial segment of $\tilde{\alpha}$ ending at $\tilde{z}_{k}^{\prime}$. Let $\tilde{\beta}^{\prime}$ be the final segment of $\tilde{\beta}$ starting at $\tilde{z}_{k}^{\prime}$. Let $\tilde{\delta}=\tilde{\alpha}^{\prime} \tilde{\beta}^{\prime} \tilde{\gamma}$.

Now $\tilde{\delta}$ is a simple closed curve in $\tilde{D}_{1}$, so by the Jordan curve theorem it must bound a disk $\tilde{B}$. Since $\gamma$ passes clockwise around $z_{i}$, there is a non-compact region to the right of $\tilde{\delta}$. Thus $\tilde{\delta}$ must pass counterclockwise around $\tilde{B}$.

Let $\alpha^{\prime}, \beta^{\prime}$ and $\delta$ be the projections of $\tilde{\alpha}^{\prime}, \tilde{\beta}^{\prime}$ and $\tilde{\delta}$ to $D_{1}$. Then $a_{i, k}-a_{i, i}$ is equal to the sum of the winding numbers of $\delta$ around each of the puncture points. This is equal to the cardinality of $\tilde{B} \cap \pi^{-1}(P)$. Since $a_{i, i}$ is maximal among all integers $a_{i^{\prime}, j^{\prime}}$, we must have $a_{i, k}=a_{i, i}$ and $\tilde{B} \cap \pi^{-1}(P)=\emptyset$. We use $\tilde{B}$ to find a digon cobounded by $\alpha^{\prime}$ and $\beta^{\prime}$.

If $\alpha^{\prime} \beta^{\prime}$ is a simple closed curve, then there is no problem. The curve $\delta=\alpha^{\prime} \beta^{\prime} \gamma$ is null-homotopic in $D \backslash P$. But $\gamma$ was chosen to be null-homotopic in $D \backslash P$. Thus $\alpha^{\prime} \beta^{\prime}$ is a null-homotopic simple closed curve, and hence bounds a disk in $D \backslash P$.

Now suppose the curve $\alpha^{\prime} \beta^{\prime}$ is not embedded. Consider the intersection of $\pi^{-1}\left(\alpha^{\prime}\right)$ with the interior of $\tilde{B}$. This is a non-empty collection of disjoint arcs, all of whose endpoints must lie on $\tilde{\beta}^{\prime}$. Let $\tilde{\alpha}^{\prime \prime}$ be an "innermost" such arc. In other words, if $\tilde{\beta}^{\prime \prime}$ is the subarc of $\tilde{\beta}^{\prime}$ with the same endpoints as $\tilde{\alpha}^{\prime \prime}$, then $\tilde{\beta}^{\prime \prime}$ intersects $\pi^{-1}\left(\alpha^{\prime}\right)$ only at its endpoints. Let $\alpha^{\prime \prime}$ and $\beta^{\prime \prime}$ be the projections of $\tilde{\alpha}^{\prime \prime}$ and $\tilde{\beta}^{\prime \prime}$ to $D \backslash P$. Then $\beta^{\prime \prime}$ meets $\alpha^{\prime}$ only at its endpoints, so $\alpha^{\prime \prime} \beta^{\prime \prime}$ is a simple closed curve. Also $\alpha^{\prime \prime} \beta^{\prime \prime}$ is null-homotopic in $D \backslash P$. Thus $\alpha^{\prime \prime} \beta^{\prime \prime}$ bounds a disk in $D \backslash P$. This disk is a digon cobounded by $\alpha^{\prime}$ and $\beta^{\prime}$.

Since $\alpha^{\prime}$ and $\beta^{\prime}$ cobound a digon, so do $T(F)$ and $N$. This contradicts the fact that $T(F)$ intersects $N$ a minimal number of times. Therefore our assumption that $b_{i, j}>b_{i, i}$ must have been false, so $b_{i, j}=b_{i, i}$.

The proof that $b_{i, j}=b_{j, j}$ is similar. This completes the proof of the claim, and hence of the Key Lemma. 


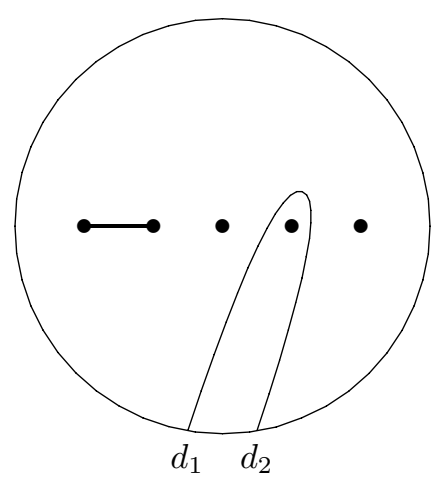

Figure 3 . The edge $E_{1}$ and the noodle $N_{4}$.

3.2. Proof of Theorem 1.1. We now use the Basic Lemma and the Key Lemma to prove that the Lawrence-Krammer representation is faithful.

Suppose $\sigma \in \mathcal{H}(D, P)$ is a homomorphism representing an element of the kernel of the Lawrence-Krammer representation. We will show that $\sigma$ is isotopic relative to $\partial D \cup P$ to the identity map.

Let $D$ be the unit disk centered at the origin in the complex plain. Let $p_{1}, \ldots, p_{n}$ lie on the real axis and satisfy $-1<p_{1}<\cdots<p_{n}<1$. Let $d_{1}$ and $d_{2}$ lie in the lower half plane, with $d_{1}$ to the left of $d_{2}$. For $i=1, \ldots, n-1$, let $E_{i}$ be the horizontal edge from $p_{i}$ to $p_{i+1}$. For $i=1, \ldots, n$, let $N_{i}$ be the noodle which winds around $p_{i}$ and no other puncture points, intersecting the real axis twice. See Figure 3

Let $F$ be a fork such that $T(F)=E_{1}$. Then $\left\langle N_{3}, F\right\rangle=0$. By the Basic Lemma, $\left\langle N_{3}, \sigma(F)\right\rangle=0$. By the Key Lemma, it follows that $\sigma\left(E_{1}\right)$ is isotopic relative to $\partial D \cup P$ to an arc which is disjoint from $N_{3}$. By composing $\sigma$ with an element of $\mathcal{I}(D, P)$ if necessary, we can assume that $\sigma\left(E_{1}\right)$ is disjoint from $N_{3}$.

Similarly, $\sigma\left(E_{1}\right)$ can be isotoped so as to be disjoint from $N_{4}$. By Lemma 3.1 this isotopy can be performed by a sequence of moves which consist of eliminating digons, and hence do not introduce any new intersections with $N_{3}$. Thus we can assume that $\sigma\left(E_{1}\right)$ is disjoint from both $N_{3}$ and $N_{4}$.

Continuing in this way, we can assume that $\sigma\left(E_{1}\right)$ is disjoint from $N_{i}$ for all $i=3, \ldots, n$. By applying one final isotopy relative to $\partial D \cup P$, we can assume that $\sigma\left(E_{1}\right)=E_{1}$ (although we have not yet eliminated the possibility that $\sigma$ reverses the orientation of $\left.E_{1}\right)$.

We can repeat the above procedure to isotope $\sigma\left(E_{2}\right)$ to $E_{2}$ while leaving $E_{1}$ fixed. Continuing in this way, we can assume that $\sigma\left(E_{i}\right)=E_{i}$ for all $i=1, \ldots, n-1$. It follows that $\sigma$ must be isotopic relative to $\partial D \cup P$ to $\left(\Delta^{2}\right)^{k}$ for some $k \in \mathbf{Z}$, where $\Delta^{2}$ is a Dehn twist about a curve which is parallel to $\partial D$.

Let $F$ be a fork with tine edge $E_{1}$ whose handle is a straight line from $d_{1}$ to $E_{1}$. One can easily check that $\left\langle N_{1}, F\right\rangle=-q$ and $\left\langle N,\left(\Delta^{2}\right)^{k}(F)\right\rangle=-q\left(q^{2 n} t^{2}\right)^{k}$. By the Basic Lemma, it follows that $k=0$, so $\sigma$ represents the trivial braid.

\section{Matrices for the LaWrence-Krammer Representation}

In this section we show how to embed $H_{2}(\tilde{C})$ into a vector space over $\mathbf{R}$. The Lawrence-Krammer representation therefore induces a faithful representation over R. We give explicit matrices for this representation. 
Theorem 4.1. Consider $\Lambda$ as a subring of $\mathbf{R}$ by assigning algebraically independent values to $q$ and $t$. Then $H_{2}(\tilde{C})$ embeds into the vector space $\mathbf{R} \otimes H_{2}(\tilde{C})$. This vector space has a basis

$$
\left\{v_{j, k}: 1 \leq j<k \leq n\right\}
$$

on which the standard generator $\sigma_{i}$ of $B_{n}$ acts as follows:

$$
\sigma_{i}\left(v_{j, k}\right)= \begin{cases}v_{j, k}, & i \notin\{j-1, j, k-1, k\}, \\ q v_{i, k}+\left(q^{2}-q\right) v_{i, j}+(1-q) v_{j, k}, & i=j-1, \\ v_{j+1, k}, & i=j \neq k-1, \\ q v_{j, i}+(1-q) v_{j, k}+\left(q^{2}-q\right) t v_{i, k}, & i=k-1 \neq j, \\ v_{j, k+1}, & i=k, \\ -t q^{2} v_{j, k}, & i=j=k-1 .\end{cases}
$$

Proof. We prove this theorem by constructing a two-complex which is homotopy equivalent to $C$. Our methods require some geometric intuition (read: "handwaving"), and some details are left to the reader.

We describe a set of generators for $\pi_{1}(C)$. For $j=1, \ldots, n$, let $\xi_{j}$ be a closed curve in $D$ based at $d_{1}$ and passing counterclockwise around $p_{j}$, and let $x_{j}$ be the arc $\left\{\xi_{j}, d_{2}\right\}$ in $C$. Let $\tau_{1}$ be an arc from $d_{1}$ to $d_{2}$, and let $\tau_{2}$ be an arc from $d_{2}$ to $d_{1}$ such that $\tau_{1} \tau_{2}$ is a simple closed curve which is oriented counterclockwise and encloses no puncture points. Let $y$ be the $\operatorname{arc}\left\{\tau_{1}, \tau_{2}\right\}$ in $C$. Let $\mathcal{G}=\left\{x_{1}, \ldots, x_{n}, y\right\}$.

We now describe some relations between these generators. For $1 \leq j \leq n$, let

$$
r_{j, j}=\left[x_{j}, y x_{j} y\right] .
$$

For $1 \leq j<k \leq n$, let

$$
r_{j, k}=\left[x_{j}, y x_{k} y^{-1}\right] .
$$

Let $\mathcal{R}=\left\{r_{j, k}: 1 \leq j \leq k \leq n\right\}$.

We will see that $\langle\mathcal{G} \mid \mathcal{R}\rangle$ is a presentation for $\pi_{1}(C)$. In fact, we will show something stronger. Let $K$ be the Cayley complex of the presentation $\langle\mathcal{G} \mid \mathcal{R}\rangle$. In other words, $K$ has one vertex, one edge for each $g \in \mathcal{G}$, and one face $f_{r}$ for each $r \in \mathcal{R}$, where $\partial f_{r}$ is attached to the 1 -skeleton according to the word $r$. We will show that $C$ is homotopy equivalent to $K$.

Let $\bar{C}$ be the set of ordered pairs of distinct points in $D \backslash P$. This is the double cover of $C$ whose fundamental group is normally generated by $x_{1}, \ldots, x_{n}$ and $y^{2}$.

Let $X_{j}=y x_{j} y^{-1}$. Let $Y=y^{2}$. Let $\overline{\mathcal{G}}=\left\{x_{1}, \ldots, x_{n}, X_{1}, \ldots, X_{n}, Y\right\}$. For $1 \leq j \leq n$, let

$$
\begin{aligned}
\bar{r}_{j, j} & =\left[x_{j}, X_{j} Y\right], \\
\bar{r}_{j, j}^{\prime} & =\left[X_{j}, Y x_{j}\right] .
\end{aligned}
$$

For $1 \leq j<k \leq n$, let

$$
\begin{aligned}
\bar{r}_{j, k} & =\left[x_{j}, X_{k}\right] \\
\bar{r}_{j, k}^{\prime} & =\left[X_{j}, Y x_{k} Y^{-1}\right] .
\end{aligned}
$$

Let

$$
\overline{\mathcal{R}}=\left\{\bar{r}_{j, k}: 1 \leq j \leq k \leq n\right\} \cup\left\{\bar{r}_{j, k}^{\prime}: 1 \leq j \leq k \leq n\right\} .
$$

Let $\bar{K}$ be the Cayley complex of $\langle\overline{\mathcal{G}} \mid \overline{\mathcal{R}}\rangle$. Then $\bar{K}$ is homotopy equivalent to the double cover of $K$ whose fundamental group is normally generated by $x_{1}, \ldots, x_{n}$ 
and $y^{2}$. To show that $C$ is homotopy equivalent to $K$, it suffices to show that $\bar{C}$ is homotopy equivalent to $\bar{K}$.

Let $\pi: \bar{C} \rightarrow D \backslash P$ be the map obtained by projection onto the first coordinate. When restricted to the interior of $\bar{C}$, this is a fiber bundle over the interior of $D \backslash P$ whose fiber is an $(n+1)$-times punctured open disk.

The base $D \backslash P$ is homotopy equivalent to a graph with one vertex and $n$ edges corresponding to $x_{1}, \ldots, x_{n}$. The fiber is homotopy equivalent to a graph with one vertex and $n+1$ edges corresponding to $X_{1}, \ldots, X_{n}$ and $Y$. The fiber bundle structure implies that $\bar{C}$ is homotopy equivalent to the Cayley complex of a presentation $\left\langle\overline{\mathcal{G}} \mid \overline{\mathcal{R}}^{\prime}\right\rangle$, where $\overline{\mathcal{R}}^{\prime}$ is a set of relations equating $Y^{x_{k}}$ and $X_{j}^{x_{k}}$ to words in $\left\{X_{1}, \ldots, X_{n}, Y\right\}$, for $j, k \in\{1, \ldots, n\}$. One can compute these relations $\overline{\mathcal{R}}^{\prime}$ by explicitly manipulating $\operatorname{arcs}$ in $\bar{C}$. They are as follows:

$$
\begin{aligned}
Y^{x_{k}} & =X_{k} Y X_{k}^{-1}, \\
X_{j}^{x_{k}} & = \begin{cases}X_{j} Y X_{j} Y^{-1} X_{j}^{-1}, & j=k, \\
X_{k} Y X_{k}^{-1} Y^{-1} X_{j} Y X_{k} Y^{-1} X_{k}^{-1}, & j<k, \\
X_{j}, & j>k .\end{cases}
\end{aligned}
$$

One can transform the relations $\overline{\mathcal{R}}^{\prime}$ to $\overline{\mathcal{R}}$ using moves which can be realized by isotopy of the attaching maps of the faces in the Cayley complex. Thus $\bar{C}$ is homotopy equivalent to $\bar{K}$, and hence $C$ is homotopy equivalent to $K$.

We are now ready to compute $H_{2}(\tilde{C})$. Let $\mathcal{C}_{1}$ and $\mathcal{C}_{2}$ be the free $\Lambda$-modules with bases $\left\{e_{g}: g \in \mathcal{G}\right\}$ and $\left\{f_{r}: r \in \mathcal{R}\right\}$ respectively. For any word $w$ in $\mathcal{G}$ we define $[w] \in \mathcal{C}_{1}$ inductively according to the rules

$$
\begin{aligned}
{[1] } & =0, \\
{[g w] } & =e_{g}+\phi(g)[w], \\
{\left[g^{-1} w\right] } & =\phi(g)^{-1}\left([w]-e_{g}\right),
\end{aligned}
$$

for any $g \in \mathcal{G}$. Then $H_{2}(\tilde{C})$ is the kernel of the map $\partial: \mathcal{C}_{2} \rightarrow \mathcal{C}_{1}$ given by $\partial f_{r}=[r]$. We compute the following:

$$
\partial f_{r}= \begin{cases}\left(1+q^{-1} t^{-1}\right)\left((1-t)\left[x_{j}\right]+(q-1)[y]\right) & \text { if } r=r_{j, j}, \\ \left(q^{-1}-q^{-2}\right)\left(-\left[x_{j}\right]+t\left[x_{k}\right]-(q-1)[y]\right) & \text { if } r=r_{j, k}, \text { where } j<k .\end{cases}
$$

Think of $\partial$ as a matrix over $\Lambda$. Then $\mathbf{R} \otimes H_{2}(\tilde{C})$ is the kernel of the same matrix thought of as a matrix over $\mathbf{R}$. It is an exercise in linear algebra to compute this. It has basis $\left\{v_{j, k}: 1 \leq j<k \leq n\right\}$, where

$$
v_{j, k}=(q-1) f_{j, j}-(q-1) t f_{k, k}+(1-t)(1+q t) f_{j, k} .
$$

Thus $H_{2}(\tilde{C})$ is the set of those $\mathbf{R}$-linear combinations of the $v_{j, k}$ which can be written as a $\Lambda$-linear combination of the $f_{r}$.

We now define certain forks $F_{j, k}$ which will correspond to the basis vectors $v_{j, k}$. Let $D$ be the unit disk centered at the origin in the complex plain. Let $p_{1}, \ldots, p_{n}$ lie on the real axis and satisfy $-1<p_{1}<\cdots<p_{n}<1$. Let $d_{1}$ and $d_{2}$ lie in the lower half plane, with $d_{1}$ to the left of $d_{2}$. For each $1 \leq j<k \leq n$, let $F_{j, k}$ be a fork which lies entirely in the closed lower half plane such that the endpoints of $T(F)$ are $p_{j}$ and $p_{k}$. Such an $F_{j, k}$ is uniquely determined up to isotopy by $j$ and $k$, and will be called a standard fork.

Let $D^{\prime} \subset D$ be a disk containing $F_{j, k}$ such that $D^{\prime} \cap P=\left\{p_{j}, p_{k}\right\}$. Let $C^{\prime}$ be the set of unordered pairs of distinct points in $D^{\prime}$. Let $\tilde{C}^{\prime}$ be the pre-image of $C^{\prime}$ in $\tilde{C}$. 
We can consider $v_{j, k}$ as an element of $H_{2}\left(\tilde{C}^{\prime}\right)$, in which case it generates $H_{2}\left(\tilde{C}^{\prime}\right)$ as a $\Lambda$-module. The surface $\tilde{\Sigma}_{2}\left(F_{j, k}\right)$ lies in $\tilde{C}^{\prime}$, so must represent the homology class $\lambda v_{j, k}$ for some $\lambda \in \Lambda$. The value of $\lambda$ does not depend on $j$ and $k$. (Actually $\lambda=1$, but we will not need this fact.)

To write $\sigma_{i}\left(v_{j, k}\right)$ in terms of basis vectors, we must find a linear combination of standard forks which represents the same element of $H_{2}(\tilde{C})$ as the fork $\sigma_{i}\left(F_{j, k}\right)$.

In the cases

- $i \notin\{j-1, j, k-1, k\}$,

- $i=j \neq k-1$, and

- $i=k$,

there is no problem because $\sigma_{i}\left(F_{j, k}\right)$ is a standard fork.

In the case $i=j=k-1$, the fork $\sigma_{i}\left(F_{j, k}\right)$ has the same tine edge as $F_{j, k}$. It follows that it represents the same surface in $\tilde{C}$, up to a change in orientation and application of a covering transformation. With some thought, or by pairing with an appropriate noodle, it is not hard to check that the correct formula is $\sigma_{i}\left(v_{j, k}\right)=-t q^{2} v_{j, k}$.

The remaining cases are $i=j-1$ and $i=k-1 \neq j$. We will use the following claim.

Claim 4.2. $\sigma_{i}\left(v_{j, k}\right)$ is a linear combination of basis vectors $v_{j^{\prime}, k^{\prime}}$ which satisfy $j^{\prime}, k^{\prime} \in\{i, i+1, j, k\}$.

Proof. There exists a disk $D^{\prime} \subset D$ such that $D^{\prime}$ contains $\sigma_{i}\left(F_{j, k}\right), D^{\prime}$ contains $F_{j^{\prime}, k^{\prime}}$ for all $j^{\prime}, k^{\prime} \in\{i, i+1, j, k\}$ with $j^{\prime}<k^{\prime}$, and $D^{\prime} \cap P=\left\{p_{i}, p_{i+1}, p_{j}, p_{k}\right\}$. Let $C^{\prime}$ be the set of unordered pairs of distinct points in $D^{\prime} \backslash P$. Let $\tilde{C}^{\prime}$ be the pre-image of $C^{\prime}$ in $\tilde{C}$. Then the vector space $\mathbf{R} \otimes H_{2}\left(\tilde{C}^{\prime}\right)$ has a basis consisting of all $v_{j^{\prime}, k^{\prime}}$ with $j^{\prime}, k^{\prime} \in\{i, i+1, j, k\}$ and $j^{\prime}<k^{\prime}$. But $\sigma\left(v_{j, k}\right)$ can be considered as an element of $H_{2}\left(\tilde{C}^{\prime}\right)$, so must be a linear combination of these basis vectors.

In the case $i=j-1$, this claim implies that $\sigma_{i}\left(F_{j, k}\right)$ represents the same element of $H_{2}(\tilde{C})$ as some linear combination of the three standard forks $F_{i, j}, F_{i, k}$, and $F_{j, k}$. By pairing with some appropriate noodles it is not hard to check that the correct linear combination is as stated in Theorem 4.1 Similar methods can be used to verify Theorem 4.1 in the last remaining case, $i=k-1 \neq j$. This completes the proof of Theorem 4.1

We conclude with some remarks on the BMW representation of braid groups, defined independently by Birman and Wenzl in BW89, and by Murakami in Mur87. V. Jones noticed a striking resemblance between the matrices described in Theorem 4.1 and those of a certain irreducible summand of the BMW representation. He asserted that the two representations should be the same after some renormalization. The details are worked out by Zinno in [Zin]. At present, there seems to be no deep explanation for this coincidence.

\section{REFERENCES}

[Big99] Stephen Bigelow, The Burau representation is not faithful for $n=5$, Geometry and Topology 3 (1999), 397-404. CMP 2000:05

[Bir74] Joan S. Birman, Braids, links, and mapping class groups, Princeton University Press, Princeton, N.J., 1974, Annals of Mathematics Studies, No. 82. MR 51:11477 (Erratum MR 54:13894) 
[BW89] Joan S. Birman and Hans Wenzl, Braids, link polynomials and a new algebra, Trans. Amer. Math. Soc. 313 (1989), no. 1, 249-273. MR 90g:57004

[Bur36] W. Burau, Über Zopfgruppen und gleichsinnig verdrillte Verkettungen, Abh. Math. Sem. Ham. II (1936), 171-178.

[FLP91] A. Fathi, F. Laudenbach, and V. Poénaru, Travaux de Thurston sur les surfaces, Société Mathématique de France, Montrouge, 1991, Séminaire Orsay, Reprint of Travaux de Thurston sur les surfaces, Soc. Math. France, Paris, 1979 [ MR 82m:57003], Astérisque No. 66-67 (1991).

[Kra99] Daan Krammer, The braid group $B_{4}$ is linear, Preprint, 1999.

[Kra00] Daan Krammer, Braid groups are linear, Preprint, 2000.

[Law90] R. J. Lawrence, Homological representations of the Hecke algebra, Comm. Math. Phys. 135 (1990), no. 1, 141-191. MR 92d:16020

[LP93] D. D. Long and M. Paton, The Burau representation is not faithful for $n \geq 6$, Topology 32 (1993), no. 2, 439-447. MR 94c:20071

[Moo91] John Atwell Moody, The Burau representation of the braid group $B_{n}$ is unfaithful for large n, Bull. Amer. Math. Soc. (N.S.) 25 (1991), no. 2, 379-384. MR 92b:20041

[Mur87] Jun Murakami, The Kauffman polynomial of links and representation theory, Osaka J. Math. 24 (1987), no. 4, 745-758. MR 89c:57007

[PR99] L. Paris and D. Rolfsen, Geometric subgroups of surface braid groups, Ann. Inst. Fourier (Grenoble) 49 (1999), no. 2, 417-472. MR 2000f:20059

[Tur] Vladimir Turaev, Faithful Linear Representations of the Braid Groups, arXiv: math.GT/0006202.

[Zin] Matthew G. Zinno, On Krammer's Representation of the Braid Group, arXiv: math.RT/0002136.

Department of Mathematics, University of Melbourne, Parkville, Victoria, AusTRALIA 3052

E-mail address: bigelow@unimelb.edu.au 